$56^{\text {ème }}$ Congrès de la SFMBCB, 02015 (2011)

DOI: $10.1051 / \mathrm{sfmbcb} / 20115602015$

(C) Owned by the authors, published by EDP Sciences, 2011

\title{
Cystadénome salivaire : à propos d'une tuméfaction jugale
}

\author{
Mathieu $A^{1,3}$, Laurence $S^{1}$, Gérard $E^{3}$, Pluot $M^{2}$, Lefèvre $B^{1}$, Hafian $H^{1}$ \\ ${ }^{1}$ Service d'Odontologie, CHU, Reims, France \\ ${ }^{2}$ Laboratoire d'Anatomie et de Cytologie pathologiques, CHU, Reims, France \\ ${ }^{3}$ Service d'Odontologie, CHR, Metz-Thionville, France \\ a-math@wanadoo.fr
}

Le cystadénome est une tumeur épithéliale rare des glandes salivaires (OMS 2005). Elle représente 4,2 à 4,7\% de l'ensemble des tumeurs bénignes (Lim et al. 2008). Histologiquement, l'aspect polykystique présente le plus souvent un épithélium d'aspect papillaire et, plus rarement, d'aspect muqueux. Il atteint préférentiellement les glandes salivaires accessoires du palais, des lèvres et des joues (Guccion et al. 1997). La parotide reste cependant une localisation fréquente (45\%). On retrouve une légère prédominance féminine (Buchner et al. 2007) et l'âge moyen de découverte est de 57 ans.

Le cas rapporté est celui d'un homme de 58 ans, adressé par son médecin traitant pour une tuméfaction jugale droite évoluant depuis 48 heures. L'interrogatoire révèle l'existence d'une masse intra-jugale asymptomatique depuis de nombreuses années. Il déclare avoir eu un traumatisme quelques heures avant l'apparition de la tuméfaction. L'examen clinique montre, une peau tendue et rouge et une muqueuse jugale normale. La palpation bidigitale est indolore. Elle met en évidence une masse intra-jugale circonscrite, mobile et de consistance molle, au contenu fluctuant. L'examen endo-buccal est sans particularité hormis la présence de débris radiculaires dans le secteur maxillaire droit. L'orthopantomogramme conforte l'examen clinique. Il n'y a pas d'adénopathies palpables. L'échographie montre une image hypoéchogéne, polylobulée, bien limitée, qui mesure $30 \mathrm{~mm}$ x $25 \mathrm{~mm}$; les échos traduisent la présence d'un contenu liquidien épais. Devant l'ensemble des éléments recueillis, l'hypothèse d'une poussée inflammatoire sur une lésion bénigne intra-jugale est retenue, probablement d'origine salivaire. La prise en charge a consisté dans un premier temps en une antibiothérapie associant amoxicilline et acide clavulanique. Après résolution de l'épisode aigu, l'exérèse chirurgicale est réalisée sous anesthésie locale potentialisée par voie d'abord endo-buccale. L'incision de la muqueuse permet de mettre en évidence une lésion kystique bilobée, de couleur jaune. Le contenu évacué lors du geste est opalin et visqueux. La lésion est adhérente au muscle buccinateur.

L'examen anatomopathologique montre une formation en majeure partie constituée par des cavités kystiques juxtaposées, de formes arrondies ou ovalaires, festonnées, contenant un abondant matériel éosinophile; certaines cavités comportent un épithélium avec des cellules internes contenant quelques vacuoles de mucus, et des cellules externes myoépithéliales. Il existe également des structures canalaires associées, festonnées, bordées par un double revêtement. Le tissu interstitiel est constitué des éléments conjonctifs physiologiques, parsemés de cellules inflammatoires mononuclées et de macrophages. L'anatomo-pathologiste conclut à un cystandénome développé sur une glande salivaire accessoire.

L'observation rapportée illustre une tuméfaction faciale aiguë secondaire à une poussée inflammatoire survenant sur un processus tumoral bénin non odontogène. En raison de sa rareté et de l'absence de caractères cliniques spécifiques, l'orientation diagnostique devant une masse intrajugale reste difficile. Les tumeurs des glandes salivaires représentent moins de $3 \%$ des tumeurs de l'extrémité céphalique et seulement $25 \%$ d'entre elles se développent sur des glandes salivaires accessoires (Dhanuthai et al. 2009). 